Pacific Journal of Mathematics

THE EQUIVALENCE OF GROUP INVARIANT POSITIVE 


\title{
THE EQUIVALENCE OF GROUP INVARIANT POSITIVE DEFINITE FUNCTIONS
}

\author{
T. R. CHOW
}

Let $G$ be a separable locally compact group; $\rho$, a positive definite function; $M(G)$, the set of all finite Radon measures; and

$$
\mathfrak{\Re}_{\rho}=\left\{\alpha \in M(G) \mid B_{\rho}(\alpha, \alpha) \equiv \int_{G \times G} \int \rho\left(t^{-1} s\right) \alpha(d s) \alpha(d t)=0\right\} .
$$

Let $H_{\rho}$ be the Hilbert space obtained by completing $M(G) / \Re_{\rho}$. Similarly define $H_{\sigma}$ as the Hilbert space corresponding to another positive definite function $\sigma, \rho$ and $\sigma$ are said to be equivalent (symbolically $\rho \sim \sigma$ ) if there is an equivalence operator $T$ from $H_{\rho}$ to $H_{\sigma}$ which is induced by the identity operator on $M(G)$; i.e. a linear homeomorphism from $H_{\rho}$ onto $H_{o}$ such that $1-T^{*} T$ is Hilbert-Schmidt. Theorem 1 and Theorem 2 give necessary and sufficient conditions for $\rho \sim \sigma$ in terms of the unitary representations of $G$ induced by $\rho$ and $\sigma$. We discuss group invariant positive definite functions on $X \times X$ where $X$ is a homogeneous space, and generalize Theorem 1 and 2 accordingly. The notion of equivalence operators comes exactly from Gaussian stochastic processes (cf. J. Feldman [4]). Some statistical applications will be discussed in a separate paper later in the year.

I. Preliminaries. Let $H$ be a separable Hilbert space; $\mathfrak{A}$, a von Neumann algebra of bounded operators in $H$. It has been proved by von Neumann that $H$ can be decomposed into a direct integral of Hilbert spaces so that $\mathfrak{U}$ is the (central) direct integral decomposition into factors (cf. von Neumann [13]). Let $\Lambda$ and $\Lambda_{1}$ be two separable metric spaces; $\mu$ and $\mu_{1}$ be two finite regular positive Borel measures on $\Lambda$ and $\Lambda_{1}$ respectively. Let

$$
H=\int_{\Lambda}^{\oplus} H(\lambda) \mu(d \lambda)
$$

and

$$
H_{1}=\int_{\Lambda_{1}}^{\oplus} H\left(\lambda_{1}\right) \mu_{1}\left(d \lambda_{1}\right)
$$

be two direct integral Hilbert spaces; $\mathfrak{A}$ and $\mathfrak{A}_{1}$ be two von Neumann algebras which have central decompositions

$$
\mathfrak{U}=\int_{\Lambda}^{\oplus} \mathfrak{U}(\lambda) \mu(d \lambda) \quad \text { and } \quad \mathfrak{U}_{1}=\int_{\Lambda_{1}}^{\oplus} \mathfrak{U}_{1}\left(\lambda_{1}\right) \mu_{1}\left(d \lambda_{1}\right)
$$


in $H$ and $H_{1}$ respectively. It can be proved (cf. J. T. Schwartz [11]) that $\mathfrak{A}$ and $\mathfrak{U}_{1}$ are spatially isomorphic if and only if there exist a pair $\tilde{\Lambda}, \tilde{\Lambda}_{1}$ of Borel subsets of $\Lambda$ and $\Lambda_{1}$ respectively such that $\mu(\Lambda-\widetilde{\Lambda})=0$ and $\mu_{1}\left(\Lambda_{1}-\widetilde{\Lambda}_{1}\right)=0$ and a Borel isomorphism $\varphi: \widetilde{\Lambda} \rightarrow \widetilde{\Lambda}_{1}$ such that $\mathfrak{U}(\lambda)$ and $\mathfrak{A}(\varphi(\lambda))$ are spatially isomorphic and such that $\mu \circ \varphi^{-1}$ is equivalent to $\mu_{1} \mid \Lambda_{1}$. Since direct integral decomposition is uniquely determined up to a set of measure zero, it can be assumed for our purpose $\Lambda=\tilde{\Lambda}$ and $\Lambda_{1}=\tilde{\Lambda}_{1}$. Hence the central decomposition is unique by the identification of $\tilde{\Lambda}$ and $\varphi(\widetilde{\Lambda})$.

For the general theory, we refer to Dixmier's book (cf. Dixmier $[1])$.

II. The equivalence of positive definite functions on groups.

Let $G$ be a separable locally compact group; $M(G)$, the set of all finite Radon measures on $G$.

1. Definition. A continuous function $\rho$ is said to be positive definite on $G$ if for any sequence of $g_{i}, i=1,2, \cdots, n$ and any sequence of complex numbers $c_{i}, i=1,2, \cdots, n$, the following is always satisfied

$$
\sum_{i, j=1}^{n} \rho\left(g_{j}^{-1} g_{i}\right) c_{i} \bar{c}_{j} \geqq 0 .
$$

It can be easily verified that $\rho$ is positive definite on $G$ if and only if

$$
\int_{G \times G} \int \rho\left(g^{-1} h\right) \mu(d h) \bar{\mu}(d g) \geqq 0
$$

for all $\mu \in M(G)$.

2. The decomposition of a positive definite function: Consider the functional $B_{r}$ on $M(G) \times M(G)$ defined by

$$
B_{i}(\alpha, \beta) \equiv \int_{G \times G} \int \gamma\left(s^{-1} t\right) \alpha(d t) \bar{\beta}(d s)
$$

where $\gamma$ is a positive definite function on $G$, and $\alpha, \beta \in M(G)$. It is clear that $\beta_{\gamma}$ is sesqui-linear and

$$
\mathfrak{U}_{\gamma}=\left\{\alpha \in M(G) \mid B_{\gamma}(\alpha, \alpha)=0\right\}
$$

is invariant under the left action of the group $G$. Let $H_{i}$ be the Hilbert space obtained by completing the quotient space $M(G) / \mathfrak{N}_{r}$ with the inner product given by (2.3). On $M(G)$, consider the linear transform defined by

$$
\widetilde{U}_{s} \alpha(A)=\alpha\left(s^{-1} A\right)
$$


where $\alpha$ is any element in $M(G)$ and $A$ is any measurable subset of $G$; and $s$, any element of $G . \mathfrak{N}_{r}$ is invariant under $\widetilde{U}_{s}$. Let $U_{s}$ be the unitary transformation on $H_{\gamma}$ which is densely defined (on $M(G) / \mathfrak{R}_{\gamma}$ ) as the quotient transformation of $\tilde{U}_{s}$. Then the pair $\left(U, H_{r}\right)$ forms a unitary representation of $G$.

Let $\mathscr{Q}$ be the von Neumann algebra generated by $\left\{U_{s}, s \in G\right\}$, that is $\left\{U_{s}, s \in G\right\}^{\prime \prime}$, the double commutant of $\left\{U_{s}, s \in G\right\}$. Let $\xi$ be the element of $H_{\gamma}$ corresponding to $\delta_{e}$, the Dirac point mass at the identity. Then

$$
\left(U_{s} \xi, \xi\right)=\int_{G \times G} \int \gamma\left(s_{1}^{-1} t\right) \delta\left(s^{-1} t\right) \delta\left(s_{1}\right) d t d s_{1}=\gamma(s) .
$$

For any $t \in G$, let

$$
\xi_{t}=U_{t} \xi
$$

Since the smallest closed linear manifold containing $\left\{\xi_{t}, t \in G\right\}$ is $H_{\gamma}$, $\mathscr{U} \xi$ is dense in $H_{r}$; so $\xi$ is cyclic. According to the theory of the central decomposition, there are a separable metric space $\Lambda$ and a Radon measure $\mu$ on $\Lambda$ such that

$$
H_{r}=\int_{\Lambda}^{\oplus} H_{r}(\lambda) \mu(d \lambda)
$$

and

$$
\mathscr{U}=\int_{\Lambda}^{\oplus} \mathscr{U}(\lambda) \mu(d \lambda)
$$

where the decomposition (2.9) is a central decomposition. It is also easy to see that

$$
U_{s}=\int_{A}^{\oplus} U_{s}(\lambda) \mu(d \lambda)
$$

where $\mu$-almost all of the $U_{s}(\lambda)$ are unitary on $H_{r}(\lambda)$ and that

$$
\xi=\int_{\Lambda}^{\oplus} \xi(\lambda) \mu(d \lambda)
$$

where $\mu$-almost all of $\xi(\lambda)$ are cyclic in their respective Hilbert spaces. $\mu$-almost all of functions $s \rightarrow\left(U_{s}(\lambda) \xi(\lambda), \xi(\lambda)\right)$, are positive definite. So $\gamma$ becomes an integral of positive definite functinns as follows:

$$
\gamma(s)=\int_{1} \gamma_{2}(s) \mu(d \lambda)
$$

where

$$
\lambda_{\lambda}(s)=\left(U_{s}(\lambda) \xi(\lambda), \xi(\lambda)\right)_{\lambda},
$$


and

$$
\gamma_{\lambda}(e)=(\xi(\lambda), \xi(\lambda))_{\lambda}=1
$$

$\mu$-almost all $\lambda$.

3. Definition. We call the measure $\mu$ normalized according to (2.12), (2.13) and $\left(2.13^{\prime}\right)$ the central Radon measure of $\gamma$ (with respect to

$$
\left.\int_{\Lambda}^{\oplus} H_{\gamma}(\lambda) \mu(d \lambda)\right) .
$$

4. The equivalence operator.

a. Definition. Let $G$ be a separable locally compact group. Two positive definite functions $\rho$ and $\sigma$ are said to be equivalent if the identity operator on $M(G)$ induces an operator $T$ on $H_{\rho}$ to $H_{\sigma}$ such that $T$ is an equalence operator (cf. introduction).

b. Let $G$ be as in definition $a ; \rho$ and $\sigma$ be two positive definite functions on $G ; H_{\rho}$ and $H_{\sigma}$ be the corresponding Hilbert spaces as defined in $\S$ II. 2. Let $\left(U, H_{\rho}\right)$ and $\left(V, H_{\sigma}\right)$ be the unitary representations of $G$ induced by $\rho$ and $\sigma$ respectively. The Dirac point mass $\delta_{e}$ at the identity of $G$ gives rise to cyclic vectors $\xi$ and $\eta$ in $H_{\rho}$ and $H_{\sigma}$ respectively. Let $\mathscr{C}=\left\{U_{s}, s \in G\right\}^{\prime \prime}$ and $\mathscr{V}^{\prime}=\left\{V_{s}, s \in G\right\}^{\prime \prime}$; and

$$
\begin{aligned}
& \mathscr{\mathscr { C }}=\int_{A}^{\oplus} \mathscr{\mathscr { C }}(\lambda) \mu(d \lambda) \\
& \mathscr{V}^{-}=\int_{\Lambda_{1}}^{\oplus} \mathscr{V}\left(\lambda_{1}\right) \mu_{1}\left(d \lambda_{1}\right)
\end{aligned}
$$

be their central decompositions.

It has been remarked in $\S I$ that if $\mathscr{C}$ and $\mathscr{Y}^{-}$are spatially isomorphic, without real loss of generality we may assume $A$ and $\Lambda_{1}$ are identical. (2.14) thus can be rewritten as

$$
\mathscr{y}^{-}=\int_{A}^{\oplus} \mathscr{C}(\lambda) \mathscr{Y}^{-}(d \lambda)
$$

if $\mathscr{U}$ and $\mathscr{V}$ are spatially isomorphic; that is if $U$ and $V$ are unitarily equivalent.

THEOREM 1. Let $G$ be a separable locally compact group; $\rho$ and $\sigma$ two positive definite functions on $G$. If $\rho$ and $\sigma$ are equivalent (symbolically $\rho \sim \sigma$ ), then

(a) $U$ and $V$ are unitarily equivalent; and

(b) With (a) permitting the existence of the central decompositions (2.14a) and $\left(2.14 \mathrm{~b}^{\prime}\right), \mu$ and $\nu$ then satisfy the following condi- 
tions, which we shall call the conditions (i), (ii) and (iii) in the rest of the discussion:

(i) $\mu$ and $\nu$ have identical nonatomic parts;

(ii) they have the same set of atoms which is countable; and

(iii) if $\mathfrak{A}$ is the set of all atoms, then $\mu(a)=\nu(a)$ unless $H_{\rho}(a)$ is finite dimensional, and

$$
\sum_{a \in \mathfrak{A}} d(a)\left(1-\frac{\mu(a)}{\nu(a)}\right)^{2}<\infty
$$

where $d(a)$ is the dimension of $H_{\rho}(a)$ if $H_{\rho}(a)$ is finite dimensional and os otherwise.

Proof. We shall divide the proof into four steps:

Step 1. To show: If $\rho \sim \sigma$, then $U$ and $V$ are unitarily equivalent. Let $\xi \in H_{\rho}$ and $\eta \in H_{\sigma}$ be the elements corresponding to $\delta_{e}$ so that

$$
\rho(s)=\left(U_{s} \xi, \xi\right)_{\rho}
$$

and

$$
\sigma(s)=\left(V_{s} \eta, \eta\right)_{\sigma}
$$

It $\rho \sim \sigma$, then it is immediately seen that $\mathfrak{R}_{\rho} \equiv \mathfrak{R}_{\sigma}$. Hence $M(G) / \mathfrak{N}_{\rho} \equiv$ $M(G) / \Re_{\sigma} \quad$ Let $T$ be the equivalence operator from $H_{\rho}$ to $H_{\sigma}$ induced by the identity operator on $M(G)$. We have for all $s \in G$

$$
T U_{s}=V_{s} T \text {. }
$$

Moreover, the center $\mathscr{Z}_{\mathscr{Z}}$ of $\mathscr{\mathscr { C }}$ is carried over by $T$ to, the center $\mathscr{L}_{\mathscr{V}}$ of $\mathscr{Y}$. Since $T$ is invertible, $T^{*}$ is well-defined on all of $H_{\sigma}$. From (2.16)

$$
U_{s} T^{*}=T^{*} V_{s}
$$

combining (2.16) and (2.17), we obtain

$$
\left(T^{*} T\right) U_{s}=U_{s}\left(T^{*} T\right) .
$$

Hence $T^{*} T$ commutes with $U_{s}$ for all $s \in G$. Consequently $T^{*} T$ is in the center $\mathscr{Z}_{\not \prime}$ of $\mathscr{\mathscr { C }}$; i.e. $T^{*} T$ is a diagonal operator (cf. Dixmier [1])

$$
T^{*} T=\int_{\Lambda}^{\oplus} a(\lambda) I(\lambda) \mu(\lambda)
$$

where $a(\lambda)$ is a nonnegative function in $L^{\infty}(\Lambda, \mu)$, and $I(\lambda)$ is the identity operator on $H_{\rho}(\lambda)$. Let $S=\left(T^{*} T\right)^{1 / 2}$, and $R$ be the unitary operator: $H_{\rho} \rightarrow H_{\sigma}$ satisfying 


$$
T=R S
$$

Then, for all $s \in G$.

$$
V_{s}=T U_{s} T^{-1}=R U_{s} R^{*}
$$

Hence $\left(V, H_{\sigma}\right)$ and $\left(U, H_{\rho}\right)$ are unitarily equivalent.

Step 2. To show: $\mu$ and $\nu$ have the same set of atoms if $\rho \sim \sigma$. If $\rho \sim \sigma$, by step $1, U$ and $V$ are unitarily equivalent so that $\mathscr{U}$ and $\mathscr{Y}^{-}$are spatially isomorphic. As we remarked in the last part of $\S I$, $\mu \sim \nu$ by the assumption of $\Lambda=\Lambda_{1}$. Since atoms are points, $\mu$ and $\nu$ therefore have the same set of atoms. This completes the step 2 .

Before we work on step 3 , we shall introduce more notations. Let $\mu_{a}$ and $\mu_{c}$ be the atomic and nonatomic parts of $\mu$ respectively; $\nu_{a}$ and $\nu_{c}$ be those of $\nu$. Let

$$
H_{\rho, c} \equiv \int_{A}^{\oplus} H_{\rho}(\lambda) \mu_{c}(d \lambda)
$$

and

$$
H_{\rho, a} \equiv \int_{\Lambda}^{\oplus} H_{\rho}(\lambda) \mu_{a}(d \lambda)
$$

Then

$$
\begin{aligned}
& H_{\rho}=H_{\rho, c} \oplus H_{\rho, a} \\
& H_{\sigma}=H_{\sigma, c} \oplus H_{\sigma, a} .
\end{aligned}
$$

It is easy to see that $T: H_{\rho, c} \rightarrow H_{\sigma, c}$ and $T: H_{\rho, c} \rightarrow H_{\sigma, a}$. If $T$ is an equivalence operator, then so are the restrictions $T \mid H_{\rho, c}$ and $T \mid H_{\rho, a}$. It is also true that $\xi=\xi_{c} \oplus \xi_{a}$ and $\eta=\eta_{a} \oplus \eta_{c}$ where $\xi_{a} \in H_{\rho, a}$, $\xi_{c} \in H_{\rho, c}, \eta_{a} \in H_{\sigma, a}$ and $\eta_{a} \in H_{\sigma, a}$ and $\eta_{c} \in H_{\sigma, c}$ are cyclic in their respective Hilbert spaces. Moreover,

$$
\left(T \mid H_{\rho, c}\right) \xi_{c}=\eta_{c}
$$

$$
\left(T \mid H_{\rho, a}\right) \xi_{a}=\eta_{a}
$$

So $\rho$ and $\sigma$ decompose into sum of two positive definite functions respectively:

$$
\begin{aligned}
& \rho \equiv \rho_{c}+\rho_{a} \equiv\left(U_{s} \xi_{c}, \xi_{c}\right)+\left(U_{s} \xi_{a}, \xi_{a}\right) \\
& \sigma \equiv \sigma_{c}+\sigma_{a} \equiv\left(V_{s} \eta_{c}, \eta_{c}\right)+\left(V_{s} \eta_{a}, \eta_{a}\right) .
\end{aligned}
$$

Step 3. To show: If $\rho \sim \sigma$, then $\mu$ and $\nu$ have identical nonatomic parts; i.e. $\mu_{c}(D)=\nu_{c}(E)$ for all measurable subsets $E$ of $\Lambda$. From above discussion, we may assume $\mu$ and $\nu$ having only nonatomic 
parts by passing from the relation $\rho \sim \sigma$ to $\rho_{c} \sim \sigma_{c}$. Suppose there exists a measurable subset $\widetilde{E}$ such that $\mu(\widetilde{E}) \neq \nu(\widetilde{E})$. Given a sufficiently small $\varepsilon>0$, there exists a measurable subset $E$ of $\widetilde{E}$ of positive measure such that

$$
\left|1-\frac{d \nu}{d \mu}(\lambda)\right|>\varepsilon
$$

for all $\lambda \in E$. Since $\mu$ is nonatomic, we can partition $E$ into a disjoint union of infinitely many measurable subsets of positive measures $\left\{E_{1}, E_{2}, \cdots\right\}$. Since $T$ is an equivalence operator, $\mu\left(E_{i}\right) \neq 0$ implies $\nu\left(E_{i}\right) \neq 0$ for all $i$. Normalizing

$$
\left\{\int_{E_{i}}^{\oplus} \xi(\lambda) \mu(d \lambda)\right\}
$$

we obtain an orthonormal set $\left\{z_{i}\right\}$. Since by (2.19), the definition of central Radon measures and $\mu \sim \nu$,

$$
\begin{aligned}
\int_{A}\|x(\lambda)\|^{2} d \nu=\left(T^{*} T x, x\right) & =\int_{A} a(\lambda)\|x(\lambda)\|^{2} d \mu \\
& =\int_{A} a(\lambda)\left(\frac{d \mu}{d \nu}\right)\|x(\lambda)\|^{2} d \nu
\end{aligned}
$$

for all $x=\int_{A}^{\oplus} x(\lambda) d \mu \in H_{p}$, it follows that

$$
a(\lambda)\left(\frac{d \mu}{d \nu}\right)(\lambda)=1 \quad \nu \text {-a.e. }
$$

i.e. $T^{*} T=\int_{A}^{\oplus}\{(d \nu) /(d \mu)\} I(x) d \mu$.

Hence

$$
\sum_{i=1}^{\infty}\left\|\left(1-T^{*} T\right) z_{i}\right\|^{2} \geqq \sum_{i=1}^{n}\left\|\left(1-T^{*} T\right) z_{i}\right\|^{2} \geqq n \varepsilon^{2} .
$$

This estimate increases to infinity as $n$ goes to infinity. This contradicts to the fact that $T$ is Hilbert-Schmidt.

Step 4. To show: If $\rho \sim \sigma$, then

$$
\sum_{\lambda \in \mathfrak{A}} d(\lambda)\left(1-\frac{\nu(\lambda)}{\mu(\lambda)}\right)^{2}<\infty
$$

As we remarked in step 3, we may reduce to the case where $\mu$ and $\nu$ have only atomic parts. The set $\mathfrak{A}$ is at most countable, for $\mu$ and $\nu$ are finite. Then 


$$
H_{\rho}=\bigoplus_{\lambda \in \mathfrak{A}} H_{\rho}(\lambda)
$$

and

$$
\|x\|^{2}=\sum_{\lambda \in \mathfrak{Q} \mathfrak{A}} \mu(\lambda)\|x(\lambda)\|_{\lambda}^{2}
$$

for all $x \in H_{\rho}$ and $x=\bigoplus_{\lambda \in \mathfrak{A}} x(\lambda)$ where $x(\lambda) \in H_{\rho}(\lambda)$. Let $\left\{\varphi_{i}\right\}$ be any orthonormal set in $H_{\rho}$. If $\rho \sim \sigma$, then

$$
T^{*} T=\bigoplus_{\lambda \in \mathfrak{Q}} a(\lambda) I(\lambda) \text {. }
$$

From a theorem of K. Fan (cf. Fan [3]), it follows that

$$
\begin{gathered}
\max _{\text {all o.N. }\left\{\varphi_{i}\right\}} \sum_{i}\left\|\left(1-T^{*} T\right) \varphi_{i}\right\|^{2}=\max \sum_{i}\left|\left(\left(1-T^{*} T\right)^{2} \varphi_{i}, \varphi_{i}\right)\right| \\
=\operatorname{Tr}\left(1-T^{*} T\right)^{2}=\sum_{A} \operatorname{Tr}\left(1-T^{*} T(\lambda)\right)^{2} .
\end{gathered}
$$

If $H_{\rho}(\lambda)$ is infinite dimensional, then $\operatorname{Tr}\left(1-T^{*} T(\lambda)\right)^{2}$ is finite only when $a(\lambda)=1$. Hence $\mu(\lambda)=\nu(\lambda)$ if $\rho \sim \sigma$ and $H_{\rho}(\lambda)$ is infinite dimensional. When $H_{\rho}(\lambda)$ is finite dimensional, then $\operatorname{Tr}\left(1-T^{*} T(\lambda)\right)^{2}=$ $d(a)(1-a(\lambda))^{2}$. Hence

$$
\begin{aligned}
\operatorname{Tr}\left(1-T^{*} T\right)^{2} & =\sum d(\lambda)(1-a(\lambda))^{2} \\
& =\sum d(\lambda)\left(1-\frac{\nu(\lambda)}{\mu(\lambda)}\right)^{2} .
\end{aligned}
$$

$1-T^{*} T$ is Hilbert-Schmidt, therefore (2.27) is finite. We have proved Theorem 1.

Theorem 1 has a converse which we shall state in the following:

THEOREM 2. If $U$ and $V$ of the last theorem are unitarily equivalent, and if the corresponding central Radon measures, $\mu$ and $\nu$ satisfy conditions (i), (ii) and (iii), then $\rho \sim \sigma$.

Proof is immediate.

III. Positive definite functions on homogeneous spaces. Let $G$ be a separable locally compact group; $H$, a closed subgroup of $G$; $X$, the space of the right cosets; $x_{0}$, be the point of $X$ which corresponds to the identity coset $H$; and finally let $M(X)$ be the set of all finite Radon measures on $X$. Then positive definite functions on $X \times X$ can be properly defined in the following way:

1. Definition. A continuous function $\hat{\rho}$ on $X \times X$ is said to be positive definite if for all $\alpha$ in $M(X)$ 


$$
\iint \hat{\rho}(x, y) \alpha(d x) \bar{\alpha}(d y) \geqq 0
$$

or alternatively,

$$
\sum_{i, j=1}^{n} \hat{\rho}\left(x_{i}, x_{j}\right) \alpha_{i} \bar{\alpha}_{j} \geqq 0
$$

for any sequence of points $x_{i}, i=1,2, \cdots, n$ and any sequence of complex numbers $c_{i}, i=1,2, \cdots, n$.

2. Definition. A positive definite function $\hat{\rho}$ is said to be $G$-invariant if, for any $g$ in $G$,

$$
\hat{\rho}(g x, g y)=\hat{\rho}(x, y) \text {. }
$$

3. Group representations and group invariant positive definite function: Let $\hat{\rho}$ be a positive definite function on $X \times X$ which is also group-invariant. Let

$$
N_{\hat{\rho}}=\left\{\alpha \in M(X) \mid \beta_{\rho}(\alpha, \alpha) \equiv \iint \hat{\rho}(x, y) \alpha(d x) \bar{\alpha}(d y)=0\right\} .
$$

Then by completing $M(X) / N_{\hat{\rho}}$, we obtain again a Hilbert space $H_{\hat{\rho}}$ with an inner product given by

$$
B_{\hat{\rho}}(\alpha, \beta) \equiv \iint \hat{\rho}(x, y) \alpha(d x) \bar{\beta}(d y) .
$$

With the group-invariance property, $N_{\hat{\rho}}$ is invariant under the action of $G$, i.e., if $\alpha \in N_{\hat{\rho}}$, then the left translates $\alpha_{g}$ of $\alpha$ also are in $N_{\hat{\rho}}$. This translation gives rise to a unitary transformation $U_{g}, g \in G$ on $H_{\hat{\rho}}$ in a similar way as in the group case (cf. §II. 2). Moreover, $\left(U, H_{\hat{\rho}}\right)$ is a unitary representation of $G$.

3. Definition. We call $\left(U, H_{\rho}\right)$ the canonical unitary representation of $G$ associated with $\hat{\rho}$.

4. Definition. Two positive definite functions $\hat{\rho}$ and $\hat{\sigma}$ on $X \times X$ are said to be equivalent if the identity operator on $M(X)$ induces an equivalence operator $\widehat{T}: H_{\hat{\rho}} \rightarrow H_{\hat{\sigma}}$.

5. Relation between positive definite functions on groups and those on homogeneous spaces: By using the same technique as used in $\S \mathrm{II}$, we may obtain the necessary and sufficient conditions for $\hat{\rho}$ and $\hat{\sigma}$ to be equivalent. However, we shall establish them through investigation of the relation between the positive definite functions on groups and those on the homogeneous spaces. 
Let $\left(U, H_{\hat{\rho}}\right)$ be the unitary representation of $G$ associated with a $G$-invariant positive definite function $\hat{\rho}$ on $X \times X$. Let $\xi \in H_{\hat{\rho}}$ be the element corresponding to $\delta_{x_{0}}$, the Dirac point mass at $x_{0}$. Then for any $s \in G$,

$$
\left(U_{s} \tilde{\xi}, \xi\right)=\int_{X \times X} \int \hat{\rho}(x, y) \delta_{s x_{0}}(d x) \delta_{x_{0}}(d y)=\hat{\rho}\left(s x_{0}, x_{0}\right) .
$$

(3.5) defines a positive definite function $\sigma$ on the group $G$ which satisfies:

$$
\rho(s)=\hat{\rho}\left(s x_{0}, x_{0}\right) .
$$

Since $x_{0}$ remains fixed under the action of $H, \rho$ satisfies

$$
\rho(s)=\rho\left(h_{1} s h_{2}\right)
$$

for all $h_{1}$ and $h_{2}$ in $H$. We thus have the following lemma.

Lemma 3.1. For any G-invariant positive definite function $\hat{\rho}$ on $X \times X$, there corresponds a positive definite function $\rho$ on $G$ which is constant on double cosets of $H$; i.e., (3.7) is satisfied.

We shall prove the following lemma before we can establish a converse form of Lemma 3.1.

Lemma 3.2. Let $\rho$ be a positive definite function on $G$. Let

$$
K=\{k \in G \mid \rho(k)=\rho(e)\},
$$

Then $K$ is a closed subgroup of $G$.

Proof. Since $\rho(e)$ is positive and finite, it can be assumed that $\rho(e)=1$. According to the theory of group representations (cf. Naimark, [10] Godement $[5,6])$ there exists a vector $\xi$ in some Hilbert space $L$ such that

$$
\rho(s)=\left(U_{s} \xi, \xi\right)
$$

where $(\cdot, \cdot)$ is the inner product in the Hilbert space $L$ and $(U, L)$ is a unitary representation of the group. Let $k \in K$. Then it can be proved that

$$
U_{k} \xi=\xi
$$

So for any $h, k \in K$

$$
U_{h} \xi=U_{k} \xi=\xi \text {. }
$$

We have $U_{k h} \xi=U_{k} U_{h} \xi=U_{k} \xi=\xi$; i.e., if $h, k \in K$, then $h k \in K$. If 
$h \in K, \rho(h)=\bar{\rho}\left(h^{-1}\right)=1=\rho\left(h^{-1}\right)$. Hence $h^{-1} \in K$ if $h \in K$. Moreover, since $\rho$ is continuous, we see that $K$ is closed.

Lemma 3.3. For any positive definite function $\rho$ on $G$, there is a largest closed subgroup $K$ such that $\rho$ is constant on double cosets of $K$. As a consequence, it gives rise to a group-invariant positive definite function $\hat{\rho}$ on $(G / K) \times(G / K)$.

Proof. Let $\{e\}$ be the subgroup consisting of only the identity of $G$. Then it is clear that $\rho$ is constant on double cosets of $\{e\}$. Let

$$
\begin{aligned}
& H=\{h \mid \rho(g h)=\rho(h g)=\rho(g) \text { for all } g \in G\} \\
& K=\{k \mid \rho(k)=\rho(e)=1\} .
\end{aligned}
$$

If $h \in H$, choosing $g=e$, then $\rho(h)=\rho(e)$. So $H \subset K$. We now prove that $\rho$ is constant on double cosets of $K$. Let $k \in K$, then as in Lemma $3.2, U_{k} \xi=\xi$ and

$$
\begin{aligned}
\rho(g k) & =\left(U_{g} U_{k} \xi, \xi\right)=\left(U_{g} \xi, \xi\right)=\rho(g)=\left(U_{g} \xi, U_{k^{-1}} \xi\right) \\
& =\left(U_{k} U_{g} \xi, \xi\right)=\rho(k g) ;
\end{aligned}
$$

i.e., $\rho(g)=\rho(K g K)$.

So $K$ is the largest closed subgroup such that $\rho$ is constant on double cosets of $K$. (3.6) defines a $G$-invariant positive definite function as it can be easily verified. Combining the preceding three lemmas, we have the following theorem.

THEOREM 3. Let $G$ be a separable locally compact group. Each positive-definite function $\rho$ on $G$ gives rise by (3.b) to a G-invariant positive definite function $\hat{\rho}$ on $(G / H) \times(G / H)$ where $H$ is the set of all $x$ in $G$ such that $\rho(x)=\rho(e)$. Conversely, to any G-invariant positive definite function $\hat{\rho}$ on $(G / H) \times(G / H)$ there corresponds by (3.6) a positive definite function $\rho$ on $G$ such that $H=\{x: \rho(x)=\rho(e)\}$.

5. Let $G$ and $H$ be the same as before; $\tau$, the canonical mapping from $G$ to $G / H=X$. Then a subset $E$ of $X$ is measurable if and only if $\tau^{-1}(E)$ is measurable in $G$ (cf. Mackey [11]). According to the theory of decomposition of measure (cf. Halmos [7, 8], von Neumann [12], Dieudonné [2], Mackey [9]), for any finite Radon measure $\alpha$ on $G$, there is a measure $\hat{\alpha}$ in $X$ such that for all measurable $E \subset X$

$$
\widehat{\alpha}(E)=\alpha\left(\tau^{-1}(E)\right)
$$

and

$$
\int_{X} f(x) \int_{G} g(s) d \alpha_{x}(s) d \tilde{\alpha}(x)=\int_{G} f(\tau(s)) g(s) d \alpha(s)
$$


where $\alpha_{x}$ is a finite Radon measure on $G$ which only lives on the coset $x$; $f$, a function in $L^{1}(X, \tilde{\alpha})$; and $g$, a bounded measurable function on $G$. Conversely, $\tilde{\alpha}$ certainly defines a measure on $G$. If $\rho$ is a positive definite function on $G$ which is constant on $H$, then $\rho\left(t^{-1} s\right)=$ $\hat{\rho}\left(s x_{0}, t x_{0}\right)=\hat{\rho}(x, y)$. By generalizing (3.11) and (3.12) to the two dimensional product measures (cf. Mackey [9]), it follows that

$$
\begin{aligned}
\int_{G \times G} \int \rho\left(t^{-1} s\right) \alpha(d s) \bar{\alpha}(d t) \\
\quad=\int_{X \times X} \int \hat{\rho}(x, y) \tilde{\alpha}(d x) \tilde{\alpha}(d y) \int_{G \times G} \int \alpha_{x}\left(d s^{\prime}\right) \alpha_{r}\left(d t^{\prime}\right) .
\end{aligned}
$$

Hence $\alpha \in \mathfrak{N}_{\rho} \subset M(G)$ if $\alpha_{x}(G)=0$. If we let $x_{\alpha} \in H_{\rho}$ be the element corresponding to $\alpha$ in $M(G)$ and $x_{\hat{\alpha}} \in H_{\hat{\rho}}$ be the element corresponding to $\alpha_{x}(G) \tilde{\alpha}$ in $M(X)$, we conclude from the above discussion that $\varphi: H_{\rho} \rightarrow H_{\hat{\rho}}$ satisfying $\varphi\left(x_{\alpha}\right)=x_{\hat{\alpha}}$ is a spatial isomorphism which commutes with the transformation induced by translations. There is a similar mapping $\psi: H_{\sigma} \rightarrow H_{\hat{\sigma}}$ satisfying $\psi\left(y_{\alpha}\right)=y_{\hat{\alpha}}$ where $y_{\alpha} \in H_{\sigma}$, $y_{\hat{\alpha}} \in H_{\hat{\sigma}}$ are the corresponding elements of $\alpha$ and $\alpha_{x}(G) \tilde{\alpha}$ respectively. Furthermore, if $T: H_{\rho} \rightarrow H_{\sigma}$ and $\widetilde{T}: H_{\hat{\rho}} \rightarrow H_{\hat{\sigma}}$ are the mapping induced by the identity mappings on $M(G)$ and $M(X)$ respectively, then it is clear that

$$
\begin{aligned}
& T x_{\alpha}=y_{\alpha} \\
& \widetilde{T} x_{\hat{\alpha}}=y_{\hat{\alpha}}
\end{aligned}
$$

and the following diagram commutes

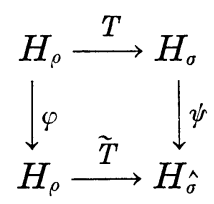

Hence $\widetilde{T}$ is a linear homeomorphism if and only if so is $T$. If $\widetilde{T}$ is such, the similar commutative diagram for $\widetilde{T}^{*}, T^{*}$ holds

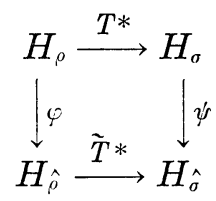

Therefore,

$$
\varphi T^{*} T=\widetilde{T}^{*} \psi T=\widetilde{T}^{*} \widetilde{T} \varphi
$$

and

$$
\left\|\left(I-T^{*} T\right) x\right\|^{2}=\left\|\varphi\left(I-T^{*} T\right) x\right\|^{2}=\left\|\left(I-\widetilde{T}^{*} \widetilde{T}\right) \varphi x\right\|^{2}
$$


We immediately conclude that $\widetilde{T}$ is an equivalence operator if and only if so is $T$, and arrive the following theorem.

THEOREM 4. Two group-invariant positive definite functions $\hat{\rho}$ and $\hat{\sigma}$ on $X=G / H$ are equivalent if and only if the corresponding positive definite functions $\rho$ and $\sigma$ on $G$ are equivalent.

6. Let $X$ be a separable metric space; $G$, a locally compact transformation group of $X$. Let $M(X)$ be the set of all finite Radon measures on $X$. The positive definite functions and group invariance property can be similarly defined as in Definitions III.1 and III.2. Suppose that $X$ has dense orbits. Let $x_{0} \in X$ such that $X_{0}=G x_{0}$ is dense in $X$. Then $X_{0}=G / H_{0}$, where $H_{0}=\left\{h \in G \mid h x_{0}=x_{0}\right\}$. We now embed $G / H_{0}$ in $X$.

Corollary 4.1. Let $X, G$ be as above; and suppose that $X$ has dense orbits. Then two G-invariant positive definite functions $\hat{\rho}$ and $\hat{\sigma}$ are equivalent if and only if the conditions in Theorem 1 are satisfied.

Proof. The elements $\xi$ in $H_{\rho}$ and $\eta$ in $H_{\sigma}$ corresponding to the Dirac point mass at $x_{0}$ are cyclic, by the continuity of positive definite functions. Hence applying Theorem 4 and Theorem 1, we assert the corollary.

Corollary 4.2. Let $X$ be a separable metris space; $G$, a locally compact group acting ergodically on $X$. Suppose that the ergodic measure $\mu$ satisfies $\mu(0)>0$ for any open set $0 \subset X$. Then $\hat{\rho} \sim \hat{\sigma}$ if and only if (a) and (b) of Theorem 1 are satisfied.

Proof. It is known that if $G$ acts ergodically on $X$ and satisfies the above hypothesis, then $X$ has dense orbits; in fact

$$
\mu\{x \mid \bar{G} \bar{x} \neq X\}=0 .
$$

Hence applying Corollary 4.1, we prove the corollary.

Thanks are due to Professor J. Feldman for his many valuable suggestions.

\section{REFERENCES}

1. J. Dixmier, Les Algebres D'operateurs dans L'espace Hilbertien, Paris, 1957.

2. J. Dieudonne, Sur le theoreme de Lebesgue-Nikodym, III, Ann. Univ. Grenoble (NS) Vol 23 (1948), 25-53. 
3. K. Fan, On a theorem of Weyl concerning eigenvalues of linear transformation I, Proc. Nat. Acad. Sci. U.S.A. 35 (1949), 652-655.

4. J. Feldman, Equivalence and perpendicularity of Gaussian Processes, Pacific J. Math. 8 699-708.

5. R. Godement, Sur la Theorie des Representations Unitaires, Ann. of Math. (2) 53 (1951), 68-124.

6. Les fonctions de type positif et la theorie des groups, Trans. Amer. Math. Soc. 63 (1948), 1-84.

7. P. R. Halmos, The decomposition of measures, Duke Math. J. 8 (1941), 386-392.

8. - On a theorem of Dieudonné, Proc. Nat. Acad. Sci. U.S.A. 35 (1949), $38-42$.

9. G. W. Mackey, Representations of locally compact groups I, Ann. of Math. 55 (1952), 101-139.

10. M. A. Naimark, Normed Rings, P. Noordhoff, Ltd., 1964.

11. J. T. Schwartz, Lecture Notes on $W^{*}$-algebras, Ch. XXI, 1962-1963, New York Univ., New York, New York.

12. J. von Neumann, Zur Operatorenmethode in der Klassischen Mechanik, Ann. of Math. 33 (1932), 789-791.

13. - On rings and operator reduction theory, Ann. of Math. 50 (1949), 401-485.

Received November 29, 1966. This work is a part of author's thesis for his $\mathrm{Ph}$. D. degree at University of California, Berkeley.

OREgON STATE UNIVERSity 


\section{PACIFIC JOURNAL OF MATHEMATICS}

\section{EDITORS}

\author{
H. ROYDEN \\ Stanford University \\ Stanford, California \\ R. R. Phelps \\ University of Washington \\ Seattle, Washington 98105
}

J. DUGUNDJI

Department of Mathematics

University of Southern California

Los Angeles, California 90007

RICHARD ARENS

University of California

Los Angeles, California 90024

\section{ASSOCIATE EDITORS}
E. F. BECKENBACH
B. H. NeumanN
F. WOLF
K. YosidA

\section{SUPPORTING INSTITUTIONS}

UNIVERSITY OF BRITISH COLUMBIA

CALIFORNIA INSTITUTE OF TECHNOLOGY

UNIVERSITY OF CALIFORNIA

MONTANA STATE UNIVERSITY

UNIVERSITY OF NEVADA

NEW MEXICO STATE UNIVERSITY

OREGON STATE UNIVERSITY

UNIVERSITY OF OREGON

OSAKA UNIVERSITY

UNIVERSITY OF SOUTHERN CALIFORNIA
STANFORD UNIVERSITY

UNIVERSITY OF TOKYO

UNIVERSITY OF UTAH

WASHINGTON STATE UNIVERSITY

UNIVERSITY OF WASHINGTON

AMERICAN MATHEMATICAL SOCIETY CHEVRON RESEARCH CORPORATION TRW SYSTEMS

NAVAL WEAPONS CENTER

Printed in Japan by International Academic Printing Co., Ltd., Tokyo, Japan 


\section{Pacific Journal of Mathematics}

\section{Vol. 26, No. $1 \quad$ November, 1968}

Efraim Pacillas Armendariz, Closure properties in radical theory......... 1

Friedrich-Wilhelm Bauer, Postnikov-decompositions of functors .......... 9

Thomas $\mathrm{Ru}-$ Wen Chow, The equivalence of group invariant positive definite

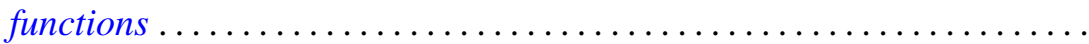

Thomas Allan Cootz, A maximum principle and geometric properties of

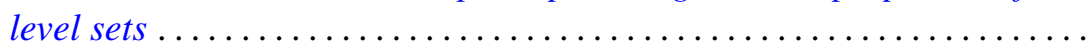

Rodolfo DeSapio, Almost diffeomorphisms of manifolds ............ 47

R. L. Duncan, Some continuity properties of the Schnirelmann density......

Ralph Jasper Faudree, Jr., Automorphism groups of finite subgroups of

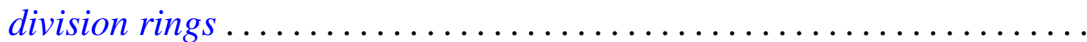

Thomas Alastair Gillespie, An invariant subspace theorem of $J$.

Feldman.........................................

George Isaac Glauberman and John Griggs Thompson, Weakly closed direct factors of Sylow subgroups .............................

Hiroshi Haruki, On inequalities generalizing a Pythagorean functional equation and Jensen's functional equation .....................

David Wilson Henderson, D-dimension. I. A new transfinite dimension.....

David Wilson Henderson, D-dimension. II. Separable spaces and

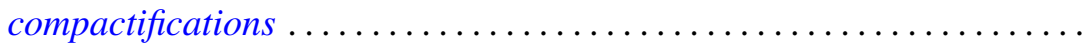

Julien O. Hennefeld, A note on the Arens products ............... 115

Richard Vincent Kadison, Strong continuity of operator functions ...

J. G. Kalbfleisch and Ralph Gordon Stanton, Maximal and minimal coverings of $(k-1)$-tuples by $k$-tuples.

Franklin Lowenthal, On generating subgroups of the Moebius group by pairs of infinitesimal transformations...

Michael Barry Marcus, Gaussian processes with stationary increments possessing discontinuous sample paths . .

Zalman Rubinstein, On a problem of Ilyeff ...

Bernard Russo, Unimodular contractions in Hilbert space. ...

David Lee Skoug, Generalized Ilstow and Feynman integrals...

William Charles Waterhouse, Dual groups of vector spaces . 